\title{
PAMPS/PVA/MMT Semi-Interpenetrating Polymer Network Hydrogel Electrolyte for Solid-State Supercapacitors
}

\author{
Jin Wang*, Hucheng Chen, Yahui Xiao, Xianghu Yu, Xiaoxuan Li \\ Anhui Province Key Laboratory of Advanced Functional Materials and Devices, School of Chemistry \\ and Chemical Engineering, Hefei University of Technology, Hefei 23009, P. R. China \\ *E-mail: jinwang@hfut.edu.cn
}

doi: $10.20964 / 2019.02 .20$

Received: 26 September 2018/ Accepted: 23 November 2018 / Published: 5 January 2019

\begin{abstract}
Polyelectrolyte hydrogels have great potential as gel-polymer electrolytes (GPE) because of their lowcost and environmental benefits. Here a polyelectrolyte hydrogel of high ionic conductivities and mechanical properties was developed via the formation of semi-interpenetrating polymer network (sIPN) GPE, which consists of Poly (2-acrylamide-2-methyl propane sulfonic acid) (PAMPS), poly(vinyl alcohol) (PVA) and sodium montmorillonite(MMT). The additions of MMT and PVA can both increase the ionic conductivities and the tensile strength of PAMPS. PAMPS/PVA/MMT s-IPN GPE show excellent overall performance of ionic conductivity of $6.4 \times 10^{-2} \mathrm{~S} \mathrm{~cm}^{-1}$ and tensile strength of $24.3 \mathrm{MPa}$ when the ratio of PVA/AMPS is at 0.8:1 and MMT is at 7wt\%. When PAMPS/PVA/MMT s-IPN is used as a GPE in supercapacitors, it exhibits good electrolyte behavior with its specific capacitance of $208 \mathrm{~F}$ $\mathrm{g}^{-1}$, close to that of using $4 \mathrm{M} \mathrm{KOH}$ aqueous solution electrolyte.
\end{abstract}

Keywords: hydrogel electrolyte, semi-interpenetrating, ionic conductivity, supercapacitors, Poly (2acrylamide-2-methyl propane sulfonic acid)

\section{FULL TEXT}

(C) 2019 The Authors. Published by ESG (www.electrochemsci.org). This article is an open access article distributed under the terms and conditions of the Creative Commons Attribution license (http://creativecommons.org/licenses/by/4.0/). 